\title{
Two-year retrospective analysis of patients undergoing direct to procedure flexible sigmoidoscopy investigation with rectal bleeding as a primary complaint
}

\section{다(1)우우}

\author{
Authors \\ Henry H. Nguyen', Robert Bechara², William G. Paterson², Lawrence C. Hookey²
}

\section{Institutions}

1 Department of Medicine, Division of Gastroenterology and Hepatology, University of Calgary, Calgary AB, Canada

2 Gastrointestinal Diseases Research Unit (GIDRU), Department of Medicine, Queen's University, Kingston ON, Canada

submitted 17.3.2017

accepted after revision 8.9.2017

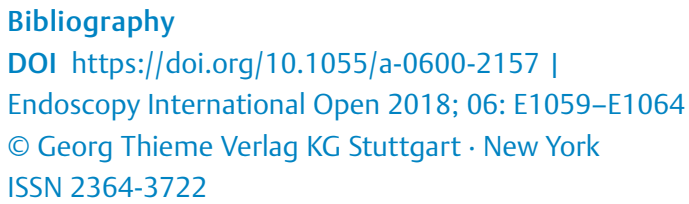

Corresponding author

Dr Lawrence Hookey, Division of Gastroenterology, Hotel Dieu Hospital, 166 Brock Street, Kingston, Ontario, Canada Fax: +1-403-592-5090

hookeyl@hdh.kari.net

\section{ABSTRACT}

Background and aims Rectal bleeding affects $\sim 15 \%$ of the general population and is a common reason for referral to gastroenterologists by primary care physicians. Direct to procedure flexible sigmoidoscopy is an appealing modality to investigate rectal bleeding due its diagnostic yield, safety profile, and accessibility. Patients referred on a routine basis for direct to procedure clinic by primary care physi- cians with the sole complaint of rectal bleeding have not previously been studied. Our study aims to explore the spectrum of diagnoses and evaluate for potential clinical predictors of underlying pathology in this specific patient population.

Methods In total, 528 charts of patients referred to the Kingston General Hospital and Hotel Dieu Hospital endoscopy units (Kingston, Canada) with the sole complaint of rectal bleeding were reviewed. All of these patients were referred on a routine basis to direct to procedure clinic from primary care physicians. The performance of various clinical variables in predicting significant pathology was assessed by univariate analysis.

Results The diagnostic spectrum of the cohort studied included hemorrhoids (75.5\%), anal fissures (4\%), ulcerative colitis (3.2\%), Crohn's disease (1.1\%), indeterminate proctitis/colitis (1.7\%), and colorectal malignancy (2.7\%). Of the various clinical variables assessed, only male sex predicted significant pathology $(25.2 \%$ of males vs $17.6 \%$ of females, $P<0.05$ ).

Conclusion Our study highlights the need for a thorough investigation of rectal bleeding given the lack of clinical predictors. Future prospective studies with more patients are needed to fully assess the utility of various clinical variables in predicting pathology in this patient population. This would allow for more effective triaging of a routine rectal bleeding, a very common reason for patient referral to gastroenterologists by primary care physicians. Flexible sigmoidoscopy was not associated with complications or missed diagnosis in our study. As such, the technique appears to be a suitable initial investigative modality for patients with rectal bleeding.

\section{Introduction}

Rectal bleeding is a problem that is reported to affect approximately $15 \%$ of the population [1]. The etiology varies widely from benign causes such as hemorrhoids, to more serious pathology such as that of inflammatory bowel disease (IBD) and lower gastrointestinal malignancy. The low predictive value of symptomatic rectal bleeding for underlying pathology, including that of malignancy [2], often leads to referral to Gastroenterology for assessment. The diagnostic yield and low complication rates of flexible sigmoidoscopy (FS) have made it an appealing modality for assessing symptomatic patients $[3,4]$. Direct to procedure clinics with flexible sigmoidoscopy (DTP-FS) have been used to address this type of referral. The lack of a sig- 
- Table 1 Primary diagnosis of patients with the primary complaint of rectal bleeding post FS $(n=528)$.

\begin{tabular}{|l|c|c|}
\hline Primary diagnosis post FS & $\begin{array}{l}\text { Frequency } \\
\text { (\# of patients) }\end{array}$ & $\begin{array}{l}\text { Percentage } \\
\text { (\%) }\end{array}$ \\
\hline Normal & 110 & 20.8 \\
\hline Hemorrhoids & 289 & 54.7 \\
\hline Anal fissures & 21 & 4 \\
\hline Adenomatous polyps & 42 & 8 \\
\hline IBD & 23 & 4.3 \\
\hline - Ulcerative colitis & 17 & 3.2 \\
\hline - Crohn's & 6 & 1.1 \\
\hline Indeterminate proctitis/colitis & 9 & 1.7 \\
\hline Rectal malignancy & 12 & 2.3 \\
\hline Colon malignancy & 1 & 0.2 \\
\hline Other & 8 & 1.7 \\
\hline Missing/no documentation & 12 & 2.3 \\
\hline FS, flexible sigmoidoscopy; IBD, inflammatory bowel disease \\
\hline
\end{tabular}

nificant difference in cancer detection between consultant led and open access endoscopy clinics [5] and the ability of FS to detect pathology in the setting of bright red blood per rectum $[6,7]$ both highlight DTP-FS clinics as an appealing option for patient referrals. However, studies have illustrated that most patients with rectal bleeding have benign underlying causes. In some studies, both malignancy and IBD have been shown to contribute to less than $\sim 5 \%$ of cases of rectal bleeds $[8,9]$. Given the predominance of benign pathology, one may question whether alternative investigative approaches to DTP-FS are more appropriate for evaluating rectal bleeding. In addition, evaluation of potential clinical features that would allow for effective and efficient triaging of these patients is also needed.

The objectives of our study were to examine the diagnostic spectrum of rectal bleeding and assess for clinical predictors of underlying gastrointestinal pathology in patients referred by primary care physicians with the primary complaint of rectal bleeding. We were specifically interested in primary care physician referred patients as they constitute the majority of referrals in our center and have not been specifically assessed as a cohort by previous studies.

\section{Methods}

This was a retrospective study of patient charts and included those who were at least 18 years of age referred from a primary care physician for rectal bleeding to be seen as a DTP-FS. These patients were assessed at either the Kingston General Hospital or Hotel Dieu Hospital endoscopy unit between June 2010 and June 2012. Both hospitals serve Kingston, Ontario and have a catchment area of 600000 in Southeastern Ontario. Patients with any of the following criteria were excluded from our study:
1) patients who were not referred from a primary care physician (e. g. Emergency Department); 2) patients whose primary complaint was not that of rectal bleeding; 3 ) patients with established gastrointestinal disorders; 4) patients with a family history of gastrointestinal malignancy; 5) patients with a previous failed/aborted FS; and 6) patients with radiation proctitis as an etiology of rectal bleeding. The exclusion criteria were established in order to specifically assess patients referred from family physicians who are routinely triaged with the primary complaint of rectal bleeding. Patients who exhibited concerning or suggestive features on history would often be triaged via alternative avenues; either on a more urgent basis or to be seen in a gastrointestinal clinic first. This study and its methodology were approved by Queen's University Health Sciences Research Ethics Board (HSREB) before initiation.

Our primary outcome of interest was that of significant pathology, namely histological features of acute and/or chronic inflammation or neoplasia. Other study variables included: 1) patient demographics; 2) duration of bleed; 3 ) descriptive quality of bleed based on history taken; 4) quality of bowel preparation; 5) need for follow-up visit or tests.

The performance of clinical variables in predicting significant pathology was assessed using univariate analysis. Two-sided $P$ values $<0.05$ were considered to be statistically significant.

\section{Results}

A total of 2059 flexible sigmoidoscopy procedure notes were reviewed with 528 charts fulfilling criteria for analysis. The median age was 51 years (range 17 to 94 years) with $54.9 \%$ female. FS was either normal or showed hemorrhoids in the majority of patients with a combined frequency of $75.5 \%$. Other findings deemed to cause the rectal bleeding included anal fissures $(4 \%)$, ulcerative colitis (3.2\%), Crohn's disease (1.1\%), indeterminate proctitis/colitis $(1.7 \%)$, and colorectal malignancy $(2.5 \%)$. Within the group of patients with colorectal malignancy, $3 / 13(23.1 \%)$ patients had a palpable mass on rectal exam and none endorsed a history of bright red blood seen only on wiping. A combined category of "other" consisted of a post-FS diagnosis of ischemic colitis, diverticulosis, or angiodysplasia with a frequency of $1.7 \%$. A total of 12 patients (2.3\%) did not have a diagnosis that was explicitly noted in the chart and thus were classified as incomplete/missing ( $\triangleright$ Table 1 ). Details on the frequency and respective percentages of duration of bleed, quality of bleed, quality of bowel preparation, and need for additional follow-up and tests are fully summarized in $>$ Table 2 . The duration of bleeding was divided into five categories; less than 1 week, 1 week to 1 month, $1-6$ months, $6-12$ months, and greater than 1 year with an overall mean duration of bleed at 68.8 weeks. On history, $13.1 \%$ and $20.1 \%$ of patients endorsed blood mixed in or coating the stool, respectively; $45.7 \%$ of patients endorsed outlet bleeding symptoms of either blood dripping into the bowl or blood noted on wiping.

In total, $20.6 \%$ and $21.8 \%$ of patients required follow-up assessment by a gastrointestinal physician and/or additional investigations (predominantly colonoscopy), respectively, after 
- Table 2 Clinical characteristics and procedural history of patients seen with the primary complaint of rectal bleeding $(n=528)$.

\begin{tabular}{|c|c|c|}
\hline Characteristics & $\begin{array}{l}\text { Frequency } \\
\text { (\# of patients) }\end{array}$ & $\begin{array}{l}\text { Percentage } \\
(\%)\end{array}$ \\
\hline \multicolumn{3}{|l|}{ Demographics } \\
\hline - Men & 238 & 45.1 \\
\hline - Women & 290 & 54.9 \\
\hline \multicolumn{3}{|l|}{ Duration of bleed } \\
\hline - Up to 1 week & 92 & 17.4 \\
\hline - >1 week up to 1 month & 70 & 13.3 \\
\hline - $>1$ month up to 6 months & 117 & 22.2 \\
\hline - $>6$ months up to 1 year & 60 & 11.4 \\
\hline - >1 year & 94 & 17.8 \\
\hline \multicolumn{3}{|l|}{ Quality of bleeding } \\
\hline - On tissue paper only & 88 & 16.7 \\
\hline - Dripping in bowl & 153 & 29.0 \\
\hline - Coating the stool & 106 & 20.1 \\
\hline - Mixed with stool & 69 & 13.1 \\
\hline \multicolumn{3}{|l|}{ Quality of bowel preparation } \\
\hline - Poor/incomplete & 69 & 13.1 \\
\hline - Moderate & 101 & 19.2 \\
\hline - Satisfactory/complete & 355 & 67.6 \\
\hline - Missing/no documentation & 3 & 0.6 \\
\hline \multicolumn{3}{|l|}{ FS completion } \\
\hline - Yes & 499 & 94.5 \\
\hline - No & 28 & 5.3 \\
\hline \multicolumn{3}{|l|}{ Need for follow-up } \\
\hline - Yes & 109 & 20.6 \\
\hline - No & 415 & 78.6 \\
\hline \multicolumn{3}{|l|}{ Need for additional tests } \\
\hline - Yes & 115 & 21.8 \\
\hline - Colonoscopy & 19 & 3.6 \\
\hline - No & 408 & 77.3 \\
\hline FS, flexible sigmoidoscopy & & \\
\hline
\end{tabular}

FS was done. Subsequent investigation with colonoscopy was performed in 19 (3.6\%) patients with rectal bleeding. Colonoscopy was carried out due to tubular adenoma(s) being detected on FS or because additional concerning gastrointestinal symptoms were elicited upon further assessment. In all of the patients who subsequently underwent colonoscopy, none had findings of proximal colonic pathology that could account for their symptoms of bright red blood per rectum.
Data analysis ( $>$ Table 3 ) found a significant association between male gender and significant pathology (25.2\% of males vs $17.6 \%$ of females, $P=0.032$ ). Age, duration of bleed, quality of rectal bleeding, and quality of bowel preparation were not predictors of significant underlying pathology.

\section{Discussion}

DTP-FS clinics are used in the assessment of rectal bleeding. Our study objectives were to assess the spectrum of diagnoses, the prevalence of significant pathology, and the performance of clinical factors in predicting significant pathology. In total, $75.5 \%$ of the primary diagnoses in our study was either that of normal FS or hemorrhoids. Polyps, malignancy, and IBD accounted for a lower percentage of $8.0 \%, 2.5 \%$, and $4.3 \%$, respectively. The proportion of patients with a benign cause of their lower gastrointestinal bleeding was quite comparable to a previous study based in Hong Kong [10]. Those authors assessed the value of FS for all patients with rectal bleeding and reported that $78.9 \%$ of patients with rectal bleeding had either a normal FS or hemorrhoids. Our study found polyp/malignancy and IBD frequencies that were higher in comparison to Choi et al. The lower rate of polyp/malignancy in the Hong Kong study may be explained by the lower FS completion rate of $81.9 \%$ compared to $94.5 \%$ in our study. An unsuccessful FS in our study was defined as an aborted exam due to poor bowel preparation or withdrawal of consent leading to an incomplete examination. The differences in IBD detection may be due to the geographical variation in prevalence of IBD, with studies demonstrating greater prevalence in North American versus Asia $[11,12]$.

Our analysis highlights that male sex was associated with significant pathology in the setting of rectal bleeding. This finding is congruent with previous studies which have highlighted a higher risk of adenoma and colorectal cancer among male sex $[13,14]$. In addition to this, the association of gender and significant pathology in our study may have been contributed by the $3.2 \%$ of patients who were found to have ulcerative colitis as a cause of their rectal bleed. It has been established that male gender is associated with ulcerative colitis [15]. Contrary to this, historical features (such as that of duration and quality of bleed), which are routinely ascertained during assessment, are not associated with serious pathology. Previous studies have echoed similar findings highlighting the low predictive value of rectal bleeding for the presence of colorectal cancer $[2,16]$ and the lack of association between quality of rectal bleeding and pathology [9]. However, it is noteworthy that a history of bright red blood just on tissue paper was not reported in any of the patients found to have colorectal cancer. Quality of bowel preparation has been shown to affect polyp detection, with poor preparations translating to missed lesions [17]. However, our study did not find any associations between quality of bowel preparation and pathology. This is likely a reflection of the use of FS instead of colonoscopy in this study. FS is a limited exam with the majority of our patients having moderate to satisfactory bowel preparations via enema, and when the preparation was unsatisfactory, the patients were administered a 
- Table 3 Univariate analysis of clinical predictors of significant pathology in patients with the primary complaint rectal bleeding $(n=528)$.

\begin{tabular}{|c|c|c|c|}
\hline \multirow[t]{2}{*}{ Characteristic } & \multicolumn{2}{|c|}{$\begin{array}{l}\text { Significant } \\
\text { pathology }\end{array}$} & \multirow[t]{2}{*}{$P$ value } \\
\hline & Yes & No & \\
\hline \multicolumn{4}{|l|}{ Demographics } \\
\hline - Age, years & 50.9 & 49.03 & 0.336 \\
\hline - Male, \% & 25.2 & 74.8 & $0.032^{1}$ \\
\hline Duration of bleed, \% & & & 0.054 \\
\hline - Up to 1 week & 17.4 & 82.6 & \\
\hline - >1 week up to 1 month & 28.6 & 71.4 & \\
\hline - $>1$ month up to 6 months & 23.9 & 76.1 & \\
\hline - $>6$ months up to 1 year & 28.3 & 71.7 & \\
\hline - >1 year & 12.8 & 87.2 & \\
\hline Quality of bleeding, \% & & & 0.580 \\
\hline - On tissue paper only & 17.0 & 83.0 & \\
\hline - Dripping in bowl & 21.6 & 78.4 & \\
\hline - Coating the stool & 22.6 & 77.4 & \\
\hline - Mixed with stool & 26.1 & 73.9 & \\
\hline Quality of bowel preparation, \% & & & 0.772 \\
\hline - Poor/incomplete & 20.3 & 79.7 & \\
\hline - Moderate & 23.8 & 76.2 & \\
\hline - Satisfactory/complete & 20.6 & 79.4 & \\
\hline FS completion, \% & & & 0.233 \\
\hline - Yes & 21.6 & 78.4 & \\
\hline - No & 10.7 & 89.3 & \\
\hline Need for follow-up, \% & & & $\mathrm{N} / \mathrm{A}$ \\
\hline - Yes & 47.7 & 52.3 & \\
\hline - No & 14.2 & 85.8 & \\
\hline Need for additional tests, \% & & & $\mathrm{N} / \mathrm{A}$ \\
\hline - Yes & 50.4 & 49.6 & \\
\hline - No & 13.0 & 87.0 & \\
\hline $\begin{array}{l}\text { FS, flexible sigmoidoscopy } \\
1 P<0.05\end{array}$ & & & \\
\hline
\end{tabular}

second enema. Thus, we were not expecting to see a difference in bowel preparation quality between those with and without pathology.

The findings of polyps and/or diverticula at FS were likely incidental rather than a true cause of blood per rectum. Given the difference in time between symptom onset and subsequent investigation with FS, it is difficult to ascertain whether diverticulosis found at FS was the cause of the bleeding that prompted referral. Given the increase in prevalence of diverticular disease with age, being estimated to occur in up to a third of patients over the age of $60[18,19]$, we suspect that this post-FS finding was most likely to be incidental. In particular, most patients presented with recurrent small volume bright red rectal bleeding with defecation, which is not typical of a diverticular bleed. Adenomatous polyps were detected in $8 \%$ of patients referred for rectal bleeding. Although this outcome is considered pathologically significant, it was also likely an incidental finding. In the vast majority of cases, adenomatous polyps are asymptomatic and rarely cause macroscopic bleeding. As such, polyps in the setting of rectal bleeding in our patients were incidental findings of significant pathology. Patients in whom we found diverticular disease and/or polyps likely had other sources of bleeding that were not identified, such as small hemorrhoids, or other outlet pathology that had healed by the time FS was performed. Proximal pathology that could account for rectal bleeding was not found in any of the patients who underwent additional testing with colonoscopy (i.e. patients with polyps). This is an interesting outcome as it suggests that FS may be a suitable first line investigative tool for outpatient rectal bleeding. The outcomes of FS can provide guidance on the need for subsequent diagnostic testing; however, additional prospective studies are needed to confirm the efficacy of FS vs other diagnostic modalities to detect pathology in patients with rectal bleeding. Interestingly, current reviews and guidelines on the management of acute overt lower gastrointestinal bleeding recommend the usage of colonoscopy as a first line investigative/ therapeutic tool. Colonoscopy after adequate hemodynamic resuscitation has been shown to give a diagnostic yield in 48 $100 \%$ of patients who present with an acute lower gastrointestinal bleed [20 - 22]. However, these recommendations are primarily designed for the management of major acute lower gastrointestinal bleeding.

There are several limitations to our current study. First, it was retrospective in design. As such, lack of a standardization in data collection for each patient may have had an effect on our analysis. The reliance on review of individual physician charting to obtain our clinical variables resulted in a lack of uniform acquisition of data points. For instance, the quality of bleeding was not consistently documented by all physicians and is estimated to have been omitted in $~ 20 \%$ of the charts. Overall, this can affect our analysis in establishing associations between clinical variables and significant pathology. Further studies utilizing a prospective and standardized approach for data collection are needed to better elucidate the utility of clinical features as predictors of pathology. Our study also assessed a very specific population of patients (exclusion criteria described above). As such, a fairly sizeable number of patients were not included in our final analysis. Patients with significant family history, past gastrointestinal history, or previous medical conditions (i.e. anemia, pelvic radiation) that predisposed them to having significant gastrointestinal pathology were not evaluated in our specific cohort of patients $[23,24]$. The majority of these patients would have been referred via an alternative pathway rather than through the routine triage pool. The purpose of our study was to assess for predictors of pathology in routine referrals such that improvements to the triaging process can be made. As such, our findings can only be applied to 
this specific group of patients (namely individuals with the sole complaint of bright red rectal bleeding and no other concerning features on history). A potential future study that can also be considered would be an assessment of clinical predictors of pathology in patients who are evaluated on a more urgent basis (direct referral to gastrointestinal clinic, referral from emergency department, urgent add on or direct to procedure).

Future research endeavors should entail the use of a prospective study design to further assess the utility of additional clinical features that would aid in elucidating the pathology of underlying gastrointestinal bleeding. In addition to the clinical parameters addressed in this study, the prospective studies should systematically assess for the presence of perianal pain, hard stools, anal pruritus/discharge, and concurrent anticoagulant use as they can all potentially aid in identifying underling pathology. In particular, the last variable is of importance as use of anticoagulation for various underlying comorbidities has been shown to increase the risk of lower gastrointestinal bleeding from various pathologies [25-27]. Finally, there is a need for a larger prospectively designed randomized control trial to assess whether FS would be an appropriate initial investigative tool for rectal bleeding compared to colonoscopy.

In conclusion, our study demonstrates that, in the setting of bright red rectal bleeding, a diagnosis of normal FS or hemorrhoids accounts for the majority of the cases. Male gender was a risk factor of significant pathology. Other clinical features including that of duration of bleed, descriptive quality of bleed, and quality of bowel preparation were not associated with significant pathology. This lack of association highlights the limited utility of clinical features in predicting pathology and underlines the need for initiating additional work-up (i.e. flexible sigmoidoscopy) to better characterize the etiology of rectal bleeding. The lack of proximal colonic findings in patients who further underwent colonoscopy, supports the use of FS as an appropriate initial modality to investigate patients with rectal bleeding.

\section{Competing interests}

Dr Henry H. Nguyen, Dr Robert Bechara, and Dr William G. Paterson do not have any declarations. Dr Lawrence C. Hookey is a consultant for Ferring Pharmaceuticals and Pentax Medical.

\section{References}

[1] Talley NJ, Jones M. Self-reported rectal bleeding in a United States community: prevalence, risk factors, and health care seeking. Am J Gastroenterol 1998; 93: 2179-2183

[2] Thompson JA, Pond CI, Ellis BG et al. Rectal bleeding in general and hospital practice; "the tip of the iceberg.". Colorectal Dis 2000; 2: 288-293

[3] Ahmad NZ, Ahmed A. Rigid or flexible sigmoidoscopy in colorectal clinics? Appraisal through a systematic review and meta-analysis J Laparoendosc Adv Surg Tech A 2012; 22: 479-487
[4] McCallum RW, Meyer CT, Marignani P et al. Flexible sigmoidoscopy: diagnostic yield in 1015 patients. Am J Gastroenterol 1984; 79: 433 437

[5] MacKenzie S, Norrie J, Vella M et al. Randomized clinical trial comparing consultant-led or open access investigation for large bowel symptoms. Br J Surg 2003; 90: 941 - 947

[6] Church JM. Analysis of the colonoscopic findings in patients with rectal bleeding according to the pattern of their presenting symptoms. Dis Colon Rectum 1991; 34: 391 - 395

[7] Shinya H, Cwern M, Wolf G. Colonoscopic diagnosis and management of rectal bleeding. Surg Clin North Am 1982; 62: 897 - 903

[8] Lee JS], Rieger NA, Stephens JH et al. Six-year prospective analysis of the rectal bleeding clinic at the Queen Elizabeth Hospital, Adelaide, South Australia. ANZ J Surg 2007; 77: $553-556$

[9] Ellis BG, Thompson MR. Factors identifying higher risk rectal bleeding in general practice. Br J Gen Pract 2005; 55: 949-955

[10] Choi HK, Law WL, Chu KW. The value of flexible sigmoidoscopy for patients with bright red rectal bleeding. Hong Kong Med J Xianggang Yi Xue Za Zhi 2003; 9: 171 - 174

[11] Molodecky NA, Soon IS, Rabi DM et al. Increasing incidence and prevalence of the inflammatory bowel diseases with time, based on systematic review. Gastroenterology 2012; 142: 46 - 54 .e42; quiz e30

[12] Ng SC, Tang W, Ching JY et al. Asia-Pacific Crohn's and Colitis Epidemiologic Study (ACCESS) Study Group. Incidence and phenotype of inflammatory bowel disease based on results from the Asia-pacific Crohn's and colitis epidemiology study. Gastroenterology 2013; 145 : $158-165$.e2

[13] Imperiale TF, Wagner DR, Lin CY et al. Risk of advanced proximal neoplasms in asymptomatic adults according to the distal colorectal findings. NEJM 2000; 343: $169-174$

[14] Schoenfeld P, Cash B, Flood A et al. CONCeRN Study Investigators. Colonoscopic screening of average-risk women for colorectal neoplasia. NEJM 2005; 352: 2061 - 2068

[15] Loftus EV, Silverstein MD, Sandborn WJ et al. Ulcerative colitis in Olmsted County, Minnesota, 1940 - 1993: incidence, prevalence, and survival. Gut 2000; 46: $336-343$

[16] Robertson R, Campbell C, Weller DP et al. Predicting colorectal cancer risk in patients with rectal bleeding. $\mathrm{Br}$ J Gen Pract 2006; 56: 763 - 767

[17] Chokshi RV, Hovis CE, Hollander T et al. Prevalence of missed adenomas in patients with inadequate bowel preparation on screening colonoscopy. Gastrointest Endosc 2012; 75: 1197-1203

[18] Parks TG. Post-mortem studies on the colon with special reference to diverticular disease. Proc R Soc Med 1968; 61: 932 - 934

[19] Peery AF, Barrett PR, Park D et al. A high-fiber diet does not protect against asymptomatic diverticulosis. Gastroenterology 2012; 142: $266-272 . e 1$

[20] Strate LL, Gralnek IM. ACG Clinical Guideline: Management of patients with acute lower gastrointestinal bleeding. Am J Gastroenterol 2016; 111: $459-474$

[21] Gralnek IM, Neeman Z, Strate LL. Acute lower gastrointestinal bleeding. NEJM 2017; 376: 1054-1063

[22] Moss AJ, Tuffaha H, Malik A. Lower GI bleeding: a review of current management, controversies and advances. Int J Colorectal Dis 2016; 31: $175-188$

[23] Imperiale TF, Ransohoff DF. Risk for colorectal cancer in persons with a family history of adenomatous polyps: a systematic review. Ann Intern Med 2012; 156: 703 - 709

[24] Olde Bekkink M, McCowan C, Falk GA et al. Diagnostic accuracy systematic review of rectal bleeding in combination with other symptoms, signs and tests in relation to colorectal cancer. $\mathrm{Br}$ J Cancer 2010; 102: $48-58$ 
[25] Lanas Á, Carrera-Lasfuentes P, Arguedas Y et al. Risk of upper and lower gastrointestinal bleeding in patients taking nonsteroidal antiinflammatory drugs, antiplatelet agents, or anticoagulants. Clin Gastroenterol Hepatol 2015; 13: 906-912.e2
[26] Lanas-Gimeno A, Lanas A. Risk of gastrointestinal bleeding during anticoagulant treatment. Expert Opin Drug Saf 2017; 16: 673-685

[27] Sherwood MW, Nessel CC, Hellkamp AS et al. Gastrointestinal bleeding in patients with atrial fibrillation treated with rivaroxaban or warfarin: ROCKET AF Trial. J Am Coll Cardiol 2015; 66: 2271-2281 